\title{
Broad-Line Profiles and Time Lags of Bloated Stars
}

\author{
Tal Alexander
}

School of Physics and Astronomy, Tel Aviv University, Tel Aviv, Israel

\begin{abstract}
The bloated stars model for AGN broad-line emission proposes that this emission originates in the mass-loss envelopes or winds of giant stars. I present here a version of the model that simultaneously reproduces typical AGN broad emission-line ratios, line profiles, and linereverberation time lags without excessive stellar collisions. This can be achieved if (1) the bloated star envelope is about 10 times denser than that of normal supergiants, (2) the fraction of the bloated stars within the stellar population falls off as $r^{-2}$, and (3) the size of the envelope is limited by the black-hole tidal forces.
\end{abstract}

\section{Introduction}

The idea that the broad-line clouds may be the mass-loss envelopes of giant stars is not new and has been studied by various authors (e.g., Edwards 1980; Mathews 1983; Scoville \& Norman 1988; Penston 1988; Kazanas 1989). This idea is motivated by the gravitational confinement of the gas, the likely existence of giant stars in AGN, and the lack of evidence for radial BLR motion in many objects, which is consistent with virialized stellar orbits. However, in spite of some promising results, the viability of the model remained unclear.

We re-investigated this idea with some new tools and a new approach (Alexander \& Netzer 1994 (AN94); 1996 (AN96); Alexander 1996 (A96)).

1. We used, for the first time, a detailed numerical model of the stellar distribution function near the black hole (Murphy, Cohn, \& Durisen 1991), which takes into account stellar collisions and links in a self-consistent way the black-hole mass, the stellar distribution function and the AGN luminosity to the AGN's age. One general result is that the stellar distribution function in the BLR volume is that of loosely bound stars on nearly parabolic orbits, since tightly bound stars are rapidly destroyed by repeated collisions.

2. We considered, for the first time, a wide range of possible envelope structures in addition to that of a normal supergiant. This is motivated by the possibility that the stellar evolution and stellar structure may be affected by the extreme conditions in the AGN. An astronomical precedent for this is the over-abundance of the very rare, high mass loss rate He I blue giants near the black hole in the Galactic center (Najarro et al. 1996). 


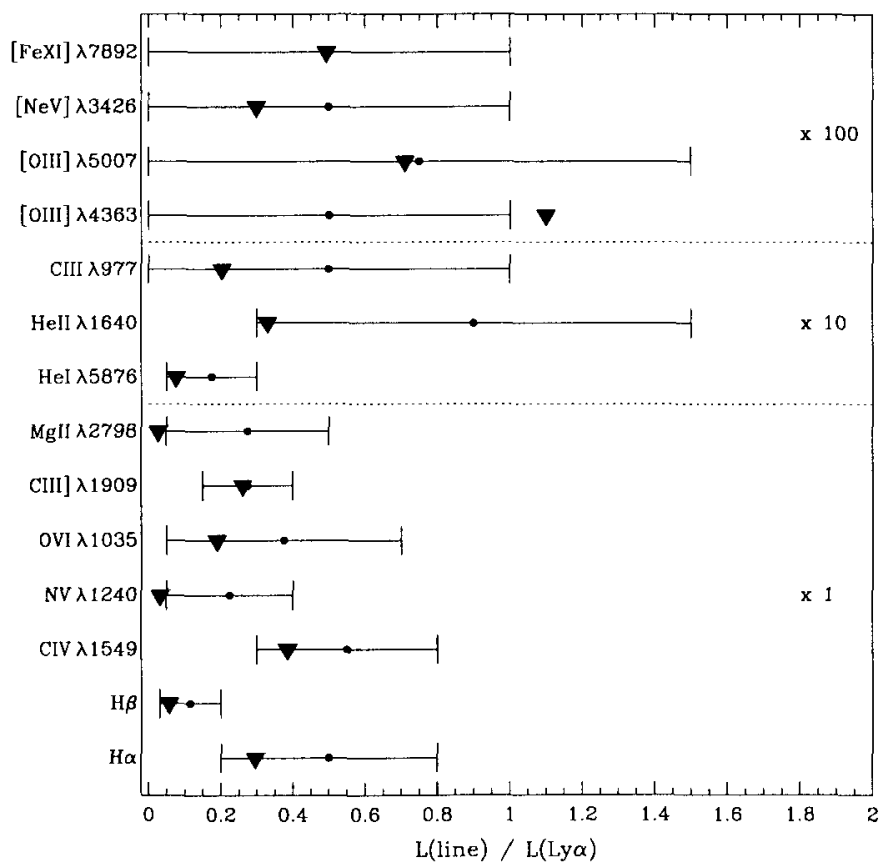

Figure 1. The broad-line luminosities (relative to $\mathrm{Ly} \alpha$ ) of the BS model (triangle). The mean observed ratios are indicated by the black dot and the ranges by the error bars (AN94).

3. We combined the cluster model and bloated-star model and used, for the first time, a full photoionization code to calculate the model predictions.

The envelope structure and size, and the physical mechanisms that determine them, are major issues in this model (AN94). It is in this respect that normal supergiants fail. Our photoionization study of their line ratios shows that their relatively thin envelopes produce unobserved strong broad forbidden lines and not enough total line emission. Since their effective cross section is small, the number of supergiants needed to reproduce the observed line luminosity is so large that they are rapidly destroyed by collisions. However, if we assume a modified supergiant wind which flows ten times slower, or that the mass loss rate is ten times higher, and that the gas is in free fall, the envelope density increases up to BLR values. The size of these objects, which we call bloated stars (BSs), is limited by the tidal forces of the black hole. With this combination of density and size, the BSs reproduce the mean observed line ratios (Fig. 1). Moreover, with their larger and denser envelopes, fewer than 100,000 such stars are needed in the BLR (less than $1 \%$ of the stellar population), and the collision rate is acceptably low (Table 1 ). 


\begin{tabular}{|c|c|}
\hline \multicolumn{2}{|l|}{ The galactic nucleus } \\
\hline AGN age & $10^{9} \mathrm{yr}$ \\
\hline Black-hole mass ${ }^{\mathbf{a}}$ & $1.9 \times 10^{8} M_{\odot}$ \\
\hline Ionizing luminosity ${ }^{a}$ & $3.6 \times 10^{44} \mathrm{erg} \mathrm{s}^{-1}$ \\
\hline Inner BLR radius ${ }^{a}$ & $0.001 \mathrm{pc}$ \\
\hline Outer BLR radius ${ }^{a}$ & $0.25 \mathrm{pc}$ \\
\hline BS to normal star ratio ${ }^{a}$ & $\propto r^{-2}$ \\
\hline \multicolumn{2}{|l|}{ The bloated star } \\
\hline Wind velocity field ${ }^{\mathbf{a}}$ & Free fall $\left(v \propto R^{-1 / 2}\right)$ \\
\hline Wind base velocity ${ }^{a}$ & $8 \times 10^{4} \mathrm{~cm} \mathrm{~s}^{-1}$ \\
\hline Wind base density & $4 \times 10^{11} \mathrm{~cm}^{-3}$ \\
\hline Wind edge density (at $30 \mathrm{ld}$ ) & $3 \times 10^{9} \mathrm{~cm}^{-3}$ \\
\hline Wind radius (at $30 \mathrm{ld}$ ) & $2 \times 10^{14} \mathrm{~cm}$ \\
\hline \multicolumn{2}{|l|}{ Other derived properties } \\
\hline BS size limiting process & Tidal disruption \\
\hline No. of BSs & $4.7 \times 10^{4}$ \\
\hline Total fraction of BSs & $2.6 \times 10^{-3}$ \\
\hline$\dot{M}_{\mathrm{evol}}$ & $0.02 M_{\odot \mathrm{yr}} \mathrm{yr}^{-1}$ \\
\hline$\dot{M}_{\mathrm{BS}}$ coll & $0.59 M_{\odot} \mathrm{yr}^{-1}$ \\
\hline$\dot{M}_{\mathrm{MS} \text { coll }}$ & $0.01 M_{\odot} \mathrm{yr}^{-1}$ \\
\hline 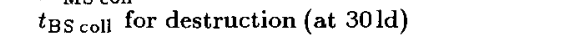 & $\sim 1.2 \times 10^{5} \mathrm{yr}$ \\
\hline Electron scattering $\dot{\tau}$ & $<0.08 \mathrm{yr}^{-1}$ \\
\hline$E_{B-V}$ & 0.1 \\
\hline Ly $\alpha$ flux-weighted radius & $25 \mathrm{ld}$ \\
\hline H $\beta$ time lag (response to PG 0844 continuum) & $60 \mathrm{~d}$ \\
\hline
\end{tabular}

\section{Results}

By modeling the observed line ratios it is possible to fix the envelope structure and the total number of BSs. In order to fix the remaining free parameter, $f_{\mathrm{BS}}$, the distribution of the BSs within the stellar population, it is necessary to turn to the line profiles. We find the model can reproduce AGN profiles if $f_{\mathrm{BS}} \propto r^{-2}$, where $r$ is the distance from the black hole. Figure 2 compares the BS profiles of a particular AGN model (Table 1) with several observed profiles taken from a large compilation of published spectra (see references in AN96). The fit to these particular observed profiles is very good. Although the sample also includes profiles that do not resemble the calculated ones, the BS model succeeds statistically in reproducing the median FWHM of the sample. It also reproduces the $F_{\lambda} \propto\left(\lambda-\lambda_{0}\right)^{-2}$ profile wings, which are seen in many AGN spectra (Penston 1988). The centrally concentrated distribution of BSs, which is necessary for reproducing the line profiles, is also consistent with the idea that the existence of these stars is linked to the unique conditions near the black hole.

Once $f_{\mathrm{BS}}$ is fixed by the observed profiles, it is possible to predict the BS model reverberation time lags and compare them to the observed relation between the BLR size and AGN luminosity (Kaspi et al. 1996). This is complicated by the fact that the observed time lag strongly depends on the variability time scale of the ionizing continuum (Netzer 1991), and the variability time scale may depend on the luminosity. In order to address this possibility, we ran through the model the observed optical light curve of PG 0804+762 (S. Kaspi, private communication), which has a luminosity similar to that of the BS model, 


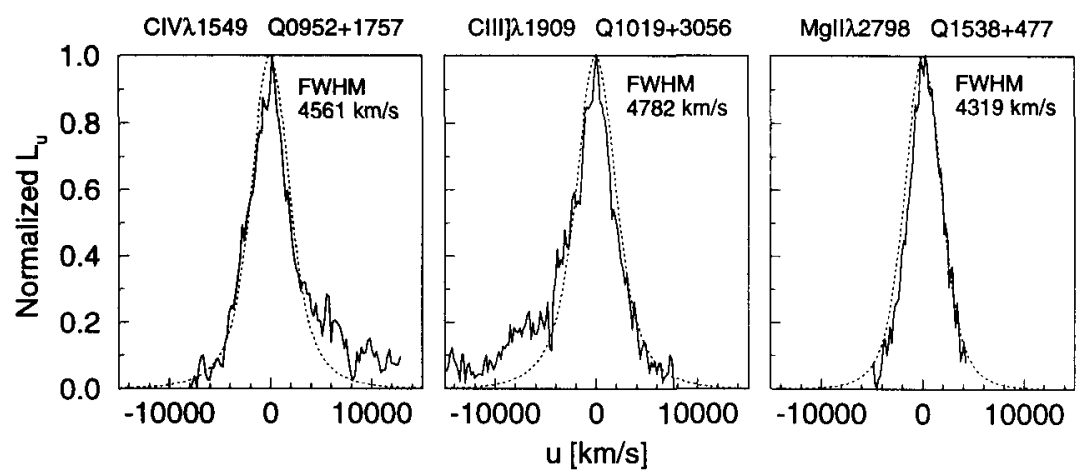

Figure 2. The observed (full line) and BS model (dotted line) profiles.

$L_{\mathrm{bol}}=4.7 \times 10^{44} \mathrm{erg} \mathrm{s}^{-1}\left(\log L_{\mathrm{bol}}=44.7\right)$. The calculated model $\mathrm{H} \beta$ time lag of $60 \mathrm{~d}\left(\log R_{\mathrm{BLR}}=1.8\right)$, fits the observed trend very well (A96, cf. Fig. 3 in Kaspi, this volume). Once again, the concentrated BS distribution is essential for reproducing the observed time lags. If, for example, we assume $f_{\mathrm{BS}}$ is constant, then the $\mathrm{H} \beta$ lag is $210 \mathrm{~d}$, which is in clear contradiction with the observations.

The version of the BS model presented here can reproduce simultaneously the three main line properties of a typical AGN: the emission-line ratios, the line profiles, and the line-reverberation time lags, while avoiding excessive stellar collisions. This is the first time any BLR model has succeeded in doing so.

Acknowledgments. I am grateful to Hagai Netzer for his close collaboration in this work and to Shai Kaspi, Mike Brotherton and Brian Murphy for their help and kind permission to use their results.

\section{References}

Alexander; T. 1996, MNRAS, submitted (A96).

Alexander, T., \& Netzer, H. 1994, MNRAS, 270, 781 (AN94).

Alexander, T., \& Netzer, H. 1996, MNRAS, submitted (AN96).

Edwards, A. C. 1980, MNRAS, 190, 757.

Kaspi, S., Smith, P.S., Maoz, D., Netzer, H., \& Jannuzi, B. T. 1996, ApJL, in press.

Kazanas, D. 1989, ApJ, 347, 74.

Mathews, W. G. 1983, ApJ, 272, 390.

Murphy, B.W., Cohn, H.N., Durisen, R.H., 1991, ApJ, 370, 60.

Najarro, F., Kudritzki, R.P., Krabbe, A., Genzel, R., Lutz, D., \& Hillier, D. J. 1996, in The Galactic Center, ed. R. Gredel, in press.

Netzer, H. 1991, in Variability of Active Galaxies, ed. W. J. Duschl, S. J. Wagner, \& M. Camerzind (Berlin: Springer), 107.

Penston, M. V. 1988, MNRAS, 233, 601.

Scoville, N., \& Norman, C. 1988, ApJ, 332, 163. 